\title{
SUB-OPTIMAL CONVERGENCE OF NON-SYMMETRIC DISCONTINUOUS GALERKIN METHODS FOR ODD POLYNOMIAL APPROXIMATIONS
}

\author{
JOHNNY GUZMÁN AND BÉATRICE RIVIÈRE
}

\begin{abstract}
We numerically verify that the non-symmetric interior penalty Galerkin method and the Oden-Babuška-Baumann method have sub-optimal convergence properties when measured in the $L^{2}$-norm for odd polynomial approximations. We provide numerical examples that use piece-wise linear and cubic polynomials to approximate a second-order elliptic problem in one and two dimensions.
\end{abstract}

\section{INTRODUCTION}

It is well known that non-symmetric discontinuous Galerkin (DG) methods for elliptic problems converge in a sub-optimal way when measured in the $L^{2}$-norm when piece-wise polynomials of even degree are used; see $[3,8]$. It was expected that odd degree polynomial approximations also converge in a sub-optimal way. The reason for this is that a non-symmetric method can produce an inconsistency error when a duality argument is used; see [2]. However, a counter-example with sub-optimal converge rates has not appeared in the literature for odd polynomial approximations. In fact, many papers reported observing optimal convergence rates when odd polynomial approximations are used; see for example [4], [3] and [1]. Moreover, two different papers prove that these method, in fact, converge in an optimal way if uniform meshes are used in one dimension; see [6] and [5]. Here we give examples of meshes in one dimension for which we clearly observe suboptimal convergence rates for both the Oden-Babuška-Baumann method [3] and the non-symmetric interior penalty Galerkin (NIPG) method [7]. Two-dimensional counter-examples can easily be constructed as well; see Section 3 .

We consider the elliptic problem on the interval $(0,1)$ :

$$
\begin{array}{rlrl}
-u^{\prime \prime}(x) & =f(x), & 0<x<1, \\
u(x) & =0, & & x=0,1 .
\end{array}
$$

In order to describe our numerical counter-example in one dimension, we need some notation. Let $0=x_{0}<x_{1}<\cdots<x_{N}=1$ be nodes of our approximation and denote this collection of points by $\mathcal{T}=\left\{x_{i}: 0 \leq i \leq N\right\}$. For a fixed integer $k \geq 1$ we define the approximation space $V(\mathcal{T})$ as

$$
V(\mathcal{T})=\left\{v \in L^{2}([0,1]):\left.v\right|_{I_{i}} \in P^{k}\left(I_{i}\right), \forall 1 \leq i \leq N\right\},
$$

where $I_{i}=\left[x_{i-1}, x_{i}\right]$ and $P^{k}\left(I_{i}\right)$ is the space of polynomials of degree at most $k$ defined on $I_{i}$. The length of the interval $I_{i}$ is denoted by $\left|I_{i}\right|$.

1991 Mathematics Subject Classification. 65M60,65N30,35L65.

Key words and phrases. finite elements, discontinuous Galerkin, suboptimal convergence rates. 
The DG approximation $U \in V(\mathcal{T})$ satisfies

$$
a(U, v)=\int_{[0,1]} f v d x, \quad \forall v \in V(\mathcal{T}),
$$

where

$$
\begin{aligned}
a(U, v):= & \sum_{i=1}^{N} \int_{I_{i}} U^{\prime} v^{\prime} d x+\sum_{i=1}^{N-1}\left(-\left\{\left\{U^{\prime}\right\}_{i} \llbracket v \rrbracket_{i}+\left\{\left\{v^{\prime}\right\}_{i} \llbracket U \rrbracket_{i}+\frac{\eta}{\bar{I}_{i}} \llbracket U \rrbracket_{i} \llbracket v \rrbracket_{i}\right)\right.\right. \\
& -U^{\prime}\left(x_{N}\right) v\left(x_{N}\right)+U^{\prime}\left(x_{0}\right) v\left(x_{0}\right)+v^{\prime}\left(x_{N}\right) U\left(x_{N}\right)-v^{\prime}\left(x_{0}\right) U\left(x_{0}\right) \\
& +\frac{\eta}{\left|I_{1}\right|} U\left(x_{0}\right) v\left(x_{0}\right)+\frac{\eta}{\left|I_{N}\right|} U\left(x_{N}\right) v\left(x_{N}\right) .
\end{aligned}
$$

Here $\bar{I}_{i}=\frac{\left|I_{i}\right|+\left|I_{i+1}\right|}{2}$ and the jumps and averages are defined as

$$
\begin{aligned}
\llbracket v \rrbracket_{i} & =v\left(x_{i}^{-}\right)-v\left(x_{i}^{+}\right) \\
\left\{\{v\}_{i}\right. & =\frac{v\left(x_{i}^{+}\right)+v\left(x_{i}^{-}\right)}{2},
\end{aligned}
$$

where

$$
v\left(x_{i}^{ \pm}\right)=\lim _{\delta \rightarrow 0^{ \pm}} v\left(x_{i}+\delta\right) .
$$

Here $\eta$ is a constant non-negative number. The Oden-Babuška-Baumann method is given if $\eta=0$ and $k \geq 2$. If $\eta>0$ we get the NIPG method. As mentioned earlier, optimal error estimates in the $L^{2}$-norm when $k$ is odd where proved in the case that the nodes $\left\{x_{i}\right\}$ are equally distributed (i.e. uniform mesh). In fact, they also proved that in this case the jumps superconverge. One might conjecture that the two are related. However, as we will see, there are many examples of meshes such that the jumps do not superconverge at the nodes, but optimal convergence rates in the $L^{2}$-norm are observed.

Meshes that produce sub-optimal approximations are meshes which result from sub-dividing each sub-interval of a uniform mesh into three sub-intervals. More precisely, let $\tilde{x}_{i}=i h$ for $i=0, \ldots, M$ where $h=\frac{1}{M}$ and $\tilde{x}_{M}=1$, then we define the nodes of our mesh as follows

$$
\begin{aligned}
x_{3 i} & =\tilde{x}_{i}, \\
x_{3 i+1} & =x_{3 i}+\alpha h, \\
x_{3 i+2} & =x_{3 i+1}+\beta h .
\end{aligned}
$$

Here $\alpha$ and $\beta$ are positive numbers that satisfy $\alpha+\beta<1$. For example, if $\alpha=$ $1 / 3=\beta$ then the resulting mesh is still uniform. However, if for example we choose $\alpha=1 / 7$ and $\beta=1 / 5$, then sub-optimal convergence rates will be observed.

It is interesting that we need to divide each sub-interval of a uniform mesh into three sub-intervals rather than two sub-intervals. Indeed, if we only divide each subinterval of a uniform mesh into two sub-intervals, then we always observe optimal convergence rates. For these type of meshes the jumps of the approximation $U$ do not superconverge at the nodes, but we still observe optimal convergence rates in the $L^{2}$-norm. 


\section{NUMERICAL EXPERIMENTS}

Although many choices of the right-hand side $f$ will give us the results we are after, we take $f$ so that the exact solution of $(1.1)$ is $u(x)=(1-x) x e^{-x^{2}}$. For the NIPG method we take $\eta=1$. However, this choice of $\eta$ is not crucial.

2.1. Piecewise linear approximations, $k=1$. In this case, the Oden-BabuškaBaumann method is not well-defined, so we only display results for the NIPG method. In Table 1 we present results for the choice $\alpha=\beta=1 / 3$ (uniform mesh) and the choice $(\alpha, \beta)=(1 / 7,1 / 5),(\alpha, \beta)=(1 / 7,1 / 7)$ and $(\alpha, \beta)=(1 / 4,1 / 2)$. We present several errors: first the error is measured in the $L^{2}$ norm. Then, a seminorm denoted by $|\cdot|_{\mathrm{J}}$ measures the jumps. Finally we compute the averages of the error at the nodes with a quantity denoted by $|\cdot|_{\mathrm{A}}$. Both $|\cdot|_{\mathrm{J}}$ and $|\cdot|_{\mathrm{A}}$ are defined as follows

$$
|u-U|_{\mathrm{J}}:=\left(\sum_{i=1}^{N-1} \bar{I}_{i} \llbracket u-U \rrbracket_{i}^{2}\right)^{1 / 2}, \quad|u-U|_{\mathrm{A}}:=\left(\sum_{i=1}^{N-1} \bar{I}_{i}\left\{\{u-U\}_{i}^{2}\right)^{1 / 2} .\right.
$$

TABLE 1. History of convergence for the NIPG with $k=1$. Three sub-intervals for each sub-interval of a uniform mesh.

\begin{tabular}{|c|c|c|c|c|c|c|c|}
\hline \multirow[b]{2}{*}{$\alpha, \beta$} & \multirow{2}{*}{$\begin{array}{c}h=\frac{1}{2^{i}} \\
i\end{array}$} & \multicolumn{2}{|c|}{$\|u-U\|_{L^{2}(\Omega)}$} & \multicolumn{2}{|c|}{$|u-U|_{\mathrm{J}}$} & \multicolumn{2}{|c|}{$|u-U|_{\mathrm{A}}$} \\
\hline & & error & order & error & order & error & order \\
\hline \multirow{8}{*}{$\frac{1}{7}, \frac{1}{5}$} & 3 & $.75 \mathrm{e}-3$ & - & $.43 \mathrm{e}-3$ & - & $.88 \mathrm{e}-3$ & - \\
\hline & 4 & $.18 \mathrm{e}-3$ & 2.06 & $.11 \mathrm{e}-3$ & 2.01 & $.24 \mathrm{e}-3$ & 1.89 \\
\hline & 5 & $0.50 \mathrm{e}-4$ & 1.86 & $.26 \mathrm{e}-4$ & 2.00 & $.72 \mathrm{e}-4$ & 1.72 \\
\hline & 6 & $.23 \mathrm{e}-4$ & 1.12 & $.66 \mathrm{e}-5$ & 2.00 & $.29 \mathrm{e}-4$ & 1.31 \\
\hline & 7 & $.13 \mathrm{e}-4$ & .85 & $.17 \mathrm{e}-5$ & 2.00 & $.14 \mathrm{e}-4$ & 1.04 \\
\hline & 8 & $.69 e-5$ & .89 & $.41 \mathrm{e}-6$ & 2.00 & $.72 \mathrm{e}-5$ & 0.98 \\
\hline & 9 & $.36 e-5$ & .94 & $.10 \mathrm{e}-6$ & 2.00 & $.36 \mathrm{e}-5$ & 0.98 \\
\hline & 10 & $.19 e-5$ & .97 & $.47 \mathrm{e}-7$ & 2.00 & $.18 \mathrm{e}-5$ & 0.99 \\
\hline \multirow{7}{*}{$\frac{1}{3}, \frac{1}{3}$} & 3 & $.66 \mathrm{e}-3$ & - & $.73 \mathrm{e}-4$ & - & $.89 \mathrm{e}-3$ & - \\
\hline & 4 & $.16 \mathrm{e}-3$ & 2.01 & $.13 \mathrm{e}-4$ & 2.52 & $.22 \mathrm{e}-3$ & 2.00 \\
\hline & 5 & $.41 \mathrm{e}-4$ & 2.01 & $.23 \mathrm{e}-5$ & 2.51 & $.56 \mathrm{e}-4$ & 2.00 \\
\hline & 6 & $.10 \mathrm{e}-4$ & 2.00 & $.40 \mathrm{e}-6$ & 2.50 & $.14 \mathrm{e}-4$ & 2.00 \\
\hline & 7 & $.25 \mathrm{e}-5$ & 2.00 & $.70 \mathrm{e}-7$ & 2.50 & $.35 \mathrm{e}-5$ & 2.00 \\
\hline & 8 & $.63 e-6$ & 2.00 & $.12 \mathrm{e}-7$ & 2.50 & $.87 \mathrm{e}-6$ & 2.00 \\
\hline & 9 & $.16 \mathrm{e}-6$ & 2.00 & $.22 \mathrm{e}-8$ & 2.50 & $.22 \mathrm{e}-6$ & 2.00 \\
\hline \multirow{7}{*}{$\frac{1}{7}, \frac{1}{7}$} & 3 & $.11 \mathrm{e}-2$ & - & $.93 \mathrm{e}-3$ & - & $.10 \mathrm{e}-2$ & - \\
\hline & 4 & $.29 \mathrm{e}-3$ & 1.91 & $.22 \mathrm{e}-3$ & 2.01 & $.28 \mathrm{e}-3$ & 1.89 \\
\hline & 5 & $.74 \mathrm{e}-4$ & 1.96 & $.57 \mathrm{e}-4$ & 2.01 & $.74 \mathrm{e}-4$ & 1.95 \\
\hline & 6 & $.19 \mathrm{e}-4$ & 1.98 & $.14 \mathrm{e}-4$ & 2.00 & $.19 \mathrm{e}-4$ & 1.97 \\
\hline & 7 & $.47 e-5$ & 1.99 & $.36 \mathrm{e}-5$ & 2.00 & $.47 e-5$ & 1.99 \\
\hline & 8 & $.12 \mathrm{e}-5$ & 1.99 & $.89 \mathrm{e}-6$ & 2.00 & $.12 \mathrm{e}-5$ & 1.99 \\
\hline & 9 & $.30 \mathrm{e}-6$ & 2.00 & $.22 \mathrm{e}-6$ & 2.00 & $.30 \mathrm{e}-6$ & 2.00 \\
\hline \multirow{7}{*}{$\frac{1}{4}, \frac{1}{2}$} & 3 & $.42 \mathrm{e}-3$ & - & $.40 \mathrm{e}-3$ & - & $.70 \mathrm{e}-3$ & - \\
\hline & 4 & $.10 \mathrm{e}-3$ & 2.01 & $.10 \mathrm{e}-3$ & 1.98 & $.17 \mathrm{e}-3$ & 2.00 \\
\hline & 5 & $.26 \mathrm{e}-4$ & 2.00 & $.26 \mathrm{e}-4$ & 1.99 & $.44 \mathrm{e}-4$ & 2.00 \\
\hline & 6 & $.66 e-5$ & 2.00 & $.64 \mathrm{e}-5$ & 1.99 & $.11 \mathrm{e}-4$ & 2.00 \\
\hline & 7 & $.16 e-5$ & 2.00 & $.16 \mathrm{e}-5$ & 2.00 & $.27 \mathrm{e}-5$ & 2.00 \\
\hline & 8 & $.41 \mathrm{e}-9$ & 2.00 & $.40 \mathrm{e}-6$ & 2.00 & $.68 \mathrm{e}-6$ & 2.00 \\
\hline & 9 & $.10 \mathrm{e}-9$ & 2.00 & $.10 \mathrm{e}-6$ & 2.00 & $.17 \mathrm{e}-6$ & 2.00 \\
\hline
\end{tabular}

We observe that the order of convergence for the $L^{2}$-norm is only one if $\alpha=$ $1 / 7, \beta=1 / 5$. Hence, this mesh produces sub-optimal approximations. Notice that 
when a uniform mesh $(\alpha=1 / 3=\beta)$ is used the $L^{2}$-norm converges with the best possible order, two. Moreover, the semi-norm of jumps superconverge with order two and $a$ half. Finally, when a non-uniform mesh given by the choice $\alpha=1 / 7=\beta$ or $\alpha=1 / 4, \beta=1 / 2$ is used optimal order of convergence is observed in the $L^{2}$ norm. However, the jumps do not superconverge. We would like to mention that in addition to the choices $\alpha=1 / 7$ and $\beta=1 / 5$, there are many more choices of $\alpha$ and $\beta$ that lead to approximations that converge in a sub-optimal way. We also mention that in all the cases the error measured in the $|u-U|_{\mathrm{A}}$ semi-norm behaves like the $L^{2}$ error.

In order to demonstrate the importance of using the above meshes to find a counter-example, we display numerical results for meshes that are obtained by subdividing each sub-interval of a uniform mesh into only two sub-intervals; see Table 2. That is,

$$
\begin{aligned}
x_{2 i} & =\tilde{x}_{i} \\
x_{2 i+1} & =x_{2 i}+\theta h,
\end{aligned}
$$

where $0<\theta<1$. As you can see, for all the choices of $\theta$ in Table 2 optimal convergence rates are observed although the jumps do not superconverge. We tried many other choices of $\theta$, and we always observe optimal convergence rates. Therefore, it was important to subdivide each sub-interval of a uniform mesh into at least three sub-intervals for sub-optimal convergence.

TABLE 2. History of convergence for the NIPG with $k=1$. Two

\begin{tabular}{|c|c|c|c|c|c|c|c|}
\hline \multirow[b]{2}{*}{$\theta$} & \multirow{2}{*}{$\begin{array}{c}h=\frac{1}{2^{i}} \\
i\end{array}$} & \multicolumn{2}{|c|}{$\|u-U\|_{L^{2}(\Omega)}$} & \multicolumn{2}{|c|}{$|u-U|_{\mathrm{J}}$} & \multicolumn{2}{|c|}{$|u-U|_{\mathrm{A}}$} \\
\hline & & error & order & error & order & error & order \\
\hline \multirow{7}{*}{$\frac{1}{3}$} & 3 & $.91 \mathrm{e}-3$ & - & $.11 \mathrm{e}-2$ & - & $.11 \mathrm{e}-2$ & - \\
\hline & 4 & $.23 \mathrm{e}-3$ & 1.95 & $.28 \mathrm{e}-3$ & 1.94 & $.29 \mathrm{e}-3$ & 1.99 \\
\hline & 5 & $0.59 \mathrm{e}-4$ & 1.98 & $.72 \mathrm{e}-4$ & 1.98 & $.73 \mathrm{e}-4$ & 1.99 \\
\hline & 6 & $.15 \mathrm{e}-4$ & 1.99 & $.18 \mathrm{e}-4$ & 1.99 & $.18 \mathrm{e}-4$ & 1.99 \\
\hline & 7 & $.37 \mathrm{e}-5$ & 1.99 & $.45 e-5$ & 2.00 & $.46 e-5$ & 2.00 \\
\hline & 8 & $.94 \mathrm{e}-6$ & 2.00 & $.11 \mathrm{e}-5$ & 2.00 & $.11 \mathrm{e}-5$ & 2.00 \\
\hline & 9 & $.23 \mathrm{e}-6$ & 2.00 & $.29 \mathrm{e}-6$ & 2.00 & $.29 \mathrm{e}-6$ & 2.00 \\
\hline \multirow{7}{*}{$\frac{1}{4}$} & 3 & $.12 \mathrm{e}-2$ & - & $.17 \mathrm{e}-2$ & - & $.11 \mathrm{e}-2$ & - \\
\hline & 4 & $.33 e-3$ & 1.89 & $.43 e-3$ & 1.95 & $.29 \mathrm{e}-3$ & 1.90 \\
\hline & 5 & $.86 \mathrm{e}-4$ & 1.95 & $.11 \mathrm{e}-3$ & 1.98 & $.76 \mathrm{e}-4$ & 1.95 \\
\hline & 6 & $.22 \mathrm{e}-4$ & 1.98 & $.27 \mathrm{e}-4$ & 1.99 & $.19 \mathrm{e}-4$ & 1.97 \\
\hline & 7 & $.54 \mathrm{e}-5$ & 1.99 & $.68 \mathrm{e}-5$ & 2.00 & $.49 \mathrm{e}-5$ & 1.99 \\
\hline & 8 & $.14 \mathrm{e}-5$ & 1.99 & $.17 \mathrm{e}-5$ & 2.00 & $.12 \mathrm{e}-5$ & 1.99 \\
\hline & 9 & $.34 \mathrm{e}-6$ & 2.00 & $.43 e-6$ & 2.00 & $.31 \mathrm{e}-6$ & 2.00 \\
\hline \multirow{7}{*}{$\frac{1}{7}$} & 3 & $.21 \mathrm{e}-2$ & - & $.24 \mathrm{e}-2$ & - & $.16 \mathrm{e}-2$ & - \\
\hline & 4 & $.57 \mathrm{e}-3$ & 1.86 & $.62 \mathrm{e}-3$ & 1.96 & $.46 \mathrm{e}-3$ & 1.81 \\
\hline & 5 & $.15 \mathrm{e}-3$ & 1.94 & $.16 \mathrm{e}-3$ & 1.98 & $.12 \mathrm{e}-3$ & 1.91 \\
\hline & 6 & $.38 \mathrm{e}-4$ & 1.97 & $.39 \mathrm{e}-4$ & 1.99 & $.31 \mathrm{e}-4$ & 1.96 \\
\hline & 7 & $.96 \mathrm{e}-5$ & 1.99 & $.98 \mathrm{e}-5$ & 2.00 & $.80 \mathrm{e}-5$ & 1.98 \\
\hline & 8 & $.24 \mathrm{e}-5$ & 1.99 & $.24 \mathrm{e}-5$ & 2.00 & $.20 \mathrm{e}-5$ & 1.99 \\
\hline & 9 & $.60 \mathrm{e}-6$ & 2.00 & $.61 \mathrm{e}-6$ & 2.00 & $.50 \mathrm{e}-6$ & 1.99 \\
\hline
\end{tabular}
sub-intervals for each sub-interval of a uniform mesh.

2.2. Piecewise cubic approximations, $k=3$. In this section we use the meshes from the previous subsection and display the errors produced by the Oden-BabuškaBaumann approximation for $k=3$. The order of convergence for the NIPG method 
are similar, so we do not present them here. Table 3 shows that the numerical rate is suboptimal for the choice $\alpha=1 / 7$ and $\beta=1 / 5$. As before we observe that the jumps do not superconverge. In the case $\alpha=\beta=1 / 7$ or $\alpha=1 / 4, \beta=1 / 2$, the convergence rates are optimal for the error in the $L^{2}$ norm, but the jumps do not superconverge. The superconvergence rate $h^{4.5}$ is obtained for the uniform case $\alpha=\beta=1 / 3$.

We now consider two subintervals and the results are given in Table 4 . For all choices of $\theta$ we obtain optimal convergence rates in the $L^{2}$ norm.

TABLE 3. History of convergence for the Oden-Babuška-Baumann method with $k=3$. Three sub-intervals for each sub-interval of a uniform mesh.

\begin{tabular}{|c|c|c|c|c|c|c|c|}
\hline \multirow[b]{2}{*}{$\alpha, \beta$} & \multirow{2}{*}{$\begin{array}{c}h=\frac{1}{2^{i}} \\
i\end{array}$} & \multicolumn{2}{|c|}{$\|u-U\|_{L^{2}(\Omega)}$} & \multicolumn{2}{|c|}{$|u-U|_{\mathrm{J}}$} & \multicolumn{2}{|c|}{$|u-U|_{\mathrm{A}}$} \\
\hline & & error & order & error & order & error & order \\
\hline$\frac{1}{7}, \frac{1}{5}$ & $\begin{array}{l}3 \\
4 \\
5 \\
6 \\
7 \\
8\end{array}$ & $\begin{array}{l}.74 \mathrm{e}-6 \\
.55 \mathrm{e}-7 \\
.57 \mathrm{e}-8 \\
.70 \mathrm{e}-9 \\
.89 \mathrm{e}-10 \\
.11 \mathrm{e}-10\end{array}$ & $\begin{array}{c}- \\
3.74 \\
3.27 \\
3.02 \\
2.97 \\
2.98\end{array}$ & $\begin{array}{l}.31 \mathrm{e}-6 \\
.19 \mathrm{e}-7 \\
.12 \mathrm{e}-8 \\
.75 \mathrm{e}-10 \\
.47 \mathrm{e}-11 \\
.29 \mathrm{e}-12\end{array}$ & $\begin{array}{c}- \\
4.00 \\
4.00 \\
4.00 \\
4.00 \\
4.00\end{array}$ & $\begin{array}{l}.40 \mathrm{e}-6 \\
.39 \mathrm{e}-7 \\
.50 \mathrm{e}-8 \\
.67 \mathrm{e}-9 \\
.88 \mathrm{e}-10 \\
.11 \mathrm{e}-10\end{array}$ & $\begin{array}{c}- \\
3.37 \\
2.96 \\
2.90 \\
2.93 \\
2.95\end{array}$ \\
\hline$\frac{1}{3}, \frac{1}{3}$ & $\begin{array}{l}3 \\
4 \\
5 \\
6 \\
7\end{array}$ & $\begin{array}{l}.79 \mathrm{e}-7 \\
.48 \mathrm{e}-8 \\
.29 \mathrm{e}-9 \\
.18 \mathrm{e}-10 \\
.12 \mathrm{e}-11\end{array}$ & $\begin{array}{c}- \\
4.03 \\
4.01 \\
4.01 \\
4.00\end{array}$ & $\begin{array}{c}.67 \mathrm{e}-8 \\
.28 \mathrm{e}-9 \\
.12 \mathrm{e}-10 \\
.52 \mathrm{e}-12 \\
.23 \mathrm{e}-13\end{array}$ & $\begin{array}{c}- \\
4.60 \\
4.54 \\
4.52 \\
4.51\end{array}$ & $\begin{array}{l}.72 \mathrm{e}-7 \\
.45 \mathrm{e}-8 \\
.28 \mathrm{e}-9 \\
.18 \mathrm{e}-10 \\
.11 \mathrm{e}-11\end{array}$ & $\begin{array}{c}- \\
3.99 \\
4.00 \\
4.00 \\
4.01\end{array}$ \\
\hline$\frac{1}{7}, \frac{1}{7}$ & $\begin{array}{l}3 \\
4 \\
5 \\
6 \\
7 \\
8\end{array}$ & $\begin{array}{l}.10 \mathrm{e}-5 \\
.62 \mathrm{e}-7 \\
.38 \mathrm{e}-8 \\
.24 \mathrm{e}-9 \\
.15 \mathrm{e}-10 \\
.95 \mathrm{e}-12\end{array}$ & $\begin{array}{c}- \\
4.02 \\
4.01 \\
4.01 \\
4.00 \\
3.96\end{array}$ & $\begin{array}{l}.35 \mathrm{e}-6 \\
.22-7 \\
.14-8 \\
.86 \mathrm{e}-10 \\
.53 \mathrm{e}-11 \\
.33 \mathrm{e}-12\end{array}$ & $\begin{array}{c}- \\
4.00 \\
4.00 \\
4.00 \\
4.00 \\
4.00\end{array}$ & $\begin{array}{l}.68 \mathrm{e}-6 \\
.44 \mathrm{e}-7 \\
.28 \mathrm{e}-8 \\
.18 \mathrm{e}-9 \\
.11 \mathrm{e}-10 \\
.79 \mathrm{e}-12\end{array}$ & $\begin{array}{c}- \\
3.94 \\
3.98 \\
3.99 \\
3.99 \\
3.82\end{array}$ \\
\hline$\frac{1}{4}, \frac{1}{2}$ & $\begin{array}{l}3 \\
4 \\
5 \\
6 \\
7\end{array}$ & $\begin{array}{l}.25 \mathrm{e}-6 \\
.15 \mathrm{e}-7 \\
.96 \mathrm{e}-9 \\
.60 \mathrm{e}-10 \\
.38 \mathrm{e}-11\end{array}$ & $\begin{array}{c}- \\
4.00 \\
4.00 \\
4.00 \\
3.99\end{array}$ & $\begin{array}{c}.15 \mathrm{e}-6 \\
.93 \mathrm{e}-8 \\
.58 \mathrm{e}-9 \\
.36 \mathrm{e}-10 \\
.22 \mathrm{e}-11\end{array}$ & $\begin{array}{c}- \\
4.01 \\
4.00 \\
4.00 \\
4.00\end{array}$ & $\begin{array}{l}.13 \mathrm{e}-6 \\
.82 \mathrm{e}-8 \\
.51 \mathrm{e}-9 \\
.32 \mathrm{e}-10 \\
.20 \mathrm{e}-11\end{array}$ & $\begin{array}{c}- \\
4.00 \\
4.00 \\
4.00 \\
3.98\end{array}$ \\
\hline
\end{tabular}

\section{Extensions to Two-Dimensional Problems}

Based on the one-dimensional counter-example, we can easily define a twodimensional counter-example on a rectangular mesh. Let the domain be the unit square $[0,1] \times[0,1]$. We let the exact solution to Laplace's equation be $u(x, y)=$ $e^{-x^{2}-y^{2}}$. On each axis, we apply the same subdivision as in the one-dimensional case. We choose the parameters $\alpha=1 / 7$ and $\beta=1 / 3$ (see Fig. 1 ). We vary the local discrete space to be $\mathbb{P}_{k}$ or the tensor product space $\mathbb{Q}_{k}$. For either spaces, we obtain sub-optimal convergence rates for the $L^{2}$ norm for the NIPG method. We note that the we get similar results for the Oden-Babuška-Baumann method. Table 3 gives the errors and rates when using the space $\mathbb{Q}_{1}$ for the NIPG method. 
TABLE 4. History of convergence for the Oden-Babuška-Baumann method with $k=3$. Two sub-intervals for each sub-interval of a uniform mesh.

\begin{tabular}{|c|c|c|c|c|c|c|c|}
\hline \multirow[b]{2}{*}{$\theta$} & \multirow{2}{*}{$\begin{array}{c}h=\frac{1}{2^{i}} \\
i\end{array}$} & \multicolumn{2}{|c|}{$\|u-U\|_{L^{2}(\Omega)}$} & \multicolumn{2}{|c|}{$|u-U|_{\mathrm{J}}$} & \multicolumn{2}{|c|}{$|u-U|_{\mathrm{A}}$} \\
\hline & & error & order & error & order & error & order \\
\hline$\frac{1}{3}$ & $\begin{array}{l}3 \\
4 \\
5 \\
6 \\
7 \\
8\end{array}$ & $\begin{array}{l}.10 \mathrm{e}-5 \\
.63 \mathrm{e}-7 \\
.39 \mathrm{e}-8 \\
.24 \mathrm{e}-9 \\
.15 \mathrm{e}-10 \\
.97 \mathrm{e}-12\end{array}$ & $\begin{array}{l}- \\
4.00 \\
4.00 \\
4.00 \\
4.00 \\
3.98\end{array}$ & $\begin{array}{l}.67 \mathrm{e}-6 \\
.44 \mathrm{e}-7 \\
.28 \mathrm{e}-8 \\
.17 \mathrm{e}-9 \\
.11 \mathrm{e}-10 \\
.68 \mathrm{e}-12\end{array}$ & $\begin{array}{l}- \\
3.95 \\
3.97 \\
4.00 \\
4.00 \\
4.00\end{array}$ & $\begin{array}{l}.44 \mathrm{e}-6 \\
.28 \mathrm{e}-7 \\
.17 \mathrm{e}-8 \\
.11 \mathrm{e}-9 \\
.69 \mathrm{e}-11 \\
.43 \mathrm{e}-12\end{array}$ & $\begin{array}{c}- \\
3.99 \\
3.99 \\
4.00 \\
4.00 \\
3.98\end{array}$ \\
\hline$\frac{1}{4}$ & $\begin{array}{l}3 \\
4 \\
5 \\
6 \\
7 \\
8\end{array}$ & $\begin{array}{l}.15 \mathrm{e}-5 \\
.92 \mathrm{e}-7 \\
.57 \mathrm{e}-8 \\
.35 \mathrm{e}-9 \\
.22 \mathrm{e}-10 \\
.14 \mathrm{e}-11\end{array}$ & $\begin{array}{c}- \\
4.01 \\
4.01 \\
4.00 \\
4.00 \\
4.00\end{array}$ & $\begin{array}{l}.89 \mathrm{e}-6 \\
.58 \mathrm{e}-7 \\
.37 \mathrm{e}-8 \\
.23 \mathrm{e}-9 \\
.14 \mathrm{e}-10 \\
.90 \mathrm{e}-12\end{array}$ & $\begin{array}{l}- \\
3.95 \\
3.98 \\
3.99 \\
4.00 \\
4.00\end{array}$ & $\begin{array}{l}.70 \mathrm{e}-6 \\
.45 \mathrm{e}-7 \\
.28 \mathrm{e}-8 \\
.18 \mathrm{e}-9 \\
.11 \mathrm{e}-10 \\
.70 \mathrm{e}-12\end{array}$ & $\begin{array}{l}- \\
3.96 \\
3.98 \\
3.99 \\
4.00 \\
3.99\end{array}$ \\
\hline$\frac{1}{7}$ & $\begin{array}{l}3 \\
4 \\
5 \\
6 \\
7 \\
8\end{array}$ & $\begin{array}{l}.22 \mathrm{e}-5 \\
.13 \mathrm{e}-6 \\
.85 \mathrm{e}-8 \\
.53 \mathrm{e}-9 \\
.33 \mathrm{e}-10 \\
.20 \mathrm{e}-11\end{array}$ & $\begin{array}{l}- \\
4.01 \\
4.01 \\
4.01 \\
4.00 \\
3.99\end{array}$ & $\begin{array}{l}.91 \mathrm{e}-6 \\
.58 \mathrm{e}-7 \\
.37 \mathrm{e}-8 \\
.23 \mathrm{e}-9 \\
.14 \mathrm{e}-10 \\
.91 \mathrm{e}-12\end{array}$ & $\begin{array}{l}- \\
3.95 \\
3.98 \\
3.99 \\
3.99 \\
4.00\end{array}$ & $\begin{array}{l}.14 \mathrm{e}-5 \\
.94 \mathrm{e}-7 \\
.60 \mathrm{e}-8 \\
.38 \mathrm{e}-9 \\
.24 \mathrm{e}-10 \\
.15 \mathrm{e}-11\end{array}$ & $\begin{array}{l}- \\
3.92 \\
3.97 \\
3.99 \\
3.99 \\
3.94\end{array}$ \\
\hline
\end{tabular}

\begin{tabular}{|c|c|c|c|c|c|c|c|c|c|c|c|}
\hline & & & प & & & & & & & & Ш \\
\hline & & & & & & & & & & & \\
\hline & & & & & & & & & & & \\
\hline & & & & & & & & & & & \\
\hline & & & & & & & & & & & \\
\hline & & & & & & & & & & & \\
\hline & & & & & & & & & & & \\
\hline & & & & & & & & & & & \\
\hline & & & & & & & & & & & 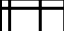 \\
\hline & & & & & & & & & & & \\
\hline & & & & & & & & & & & \\
\hline & & & & & & & & & & & \\
\hline & & & & & & & & & & & \\
\hline & & & ] & & & & & & & & \\
\hline & & & & & & & & & & & 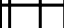 \\
\hline & & & D & & & & . & & & & \\
\hline
\end{tabular}

FIGURE 1. Example of non-uniform two dimensional mesh with $h=1 / 8, \alpha=1 / 7$ and $\beta=1 / 3$

\section{Concluding Remarks}

In this paper we showed numerically that the NIPG method and the OdenBabuška-Baumann method have sub-optimal convergence properties for odd polynomial approximations. To the best or our knowledge, this is the first time that an example has been presented that clearly demonstrates sub-optimal convergence. 
TABLE 5. History of convergence for the NIPG method using the local space $\mathbb{Q}_{1}$. On each axis there are three sub-intervals for each sub-interval of a uniform mesh.

\begin{tabular}{|c|c|cc|}
\hline & $h=\frac{1}{2^{i}}$ & \multicolumn{2}{|l|}{$\|u-U\|_{L^{2}(\Omega)}$} \\
$\alpha, \beta$ & $i$ & error & order \\
\hline \multirow{5}{*}{$\frac{1}{7}, \frac{1}{3}$} & 3 & $.28 \mathrm{e}-3$ & - \\
& 4 & $.76 \mathrm{e}-4$ & 1.89 \\
& 5 & $.23 \mathrm{e}-4$ & 1.70 \\
& 6 & $.87 \mathrm{e}-5$ & 1.40 \\
& 7 & $.38 \mathrm{e}-5$ & 1.16 \\
\hline
\end{tabular}

\section{Acknowledgments}

The authors would like to thank Bernardo Cockburn for many fruitful discussions.

\section{REFERENCES}

[1] P. Antonietti and L. Heltai, Numerical validation of a class of mixed discontinuous Galerkin methods for Darcy flow, Comput. Methods Appl. Mech. Engrg. 196 (2007), 4505-4520.

[2] D. N. Arnold, F. Brezzi, B. Cockburn, and L. D. Marini, Unified analysis of discontinuous Galerkin methods for elliptic problems, SIAM J. Numer. Anal. 39 (2002), 1749-1779.

[3] J. Oden, I. Babuška and C. Baumann, A discontinuous hp finite element method for diffusion problems, J. Comput. Phys. 146 (1998), 491-519.

[4] P. Castillo, Performance of discontinuous Galerkin methods for elliptic PDE's, SIAM J. Sci. Comput. 24 (2002), 524-547.

[5] H. Chen, Superconvergence properties of discontinuous Galerkin methods for two-point boundary value problems, Int. J. Numer. Anal. Model. 3 (2006), 163-185.

[6] M. Larson and J. Niklasson, Analysis of a family of discontinuous Galerkin methods for elliptic problems: the one dimensional case, Numer. Math. 99 (2004), 113-130.

[7] B. Rivière, M. Wheeler and V. Girault, A priori error estimates for finite element methods based on discontinuous approximation spaces for elliptic problems, SIAM J. Numer. Anal. 39 (2001), 902-931.

[8] B. Rivière, M. Wheeler and K. Banas, Part II. Discontinuous Galerkin method applied to a single phase flow in porous media, Computational Geosciences 4 (2000), 337-349.

Division of Applied Mathematics, Brown University, Providence, Ri 02906, USA.

E-mail address: johnny_guzman@brown.edu

Department of Computational and Applied Mathematics, Rice University, Houston, TX 77005 , USA.

E-mail address: riviere@caam.rice.edu 